\title{
Stenotic aortic valves have dysfunctional mechanisms of anti-inflammation: Implications for aortic stenosis
}

Joon H. Lee, MD, Xianzhong Meng, MD, PhD, Michael J. Weyant, MD, T. Brett Reece, MD, Joseph C. Cleveland, Jr, MD, and David A. Fullerton, MD

\begin{abstract}
Objective: Aortic stenosis is an inflammatory disease, associated with increased tissue levels of interleukin-1 beta. We hypothesized that the antagonist of interleukin-1 beta, interleukin-1 receptor antagonist, is deficient in aortic valves and that its production by aortic valve interstitial cells is less in cells from stenotic valves than from controls.

Methods: Valve leaflets from stenotic aortic valves $(n=6)$ and from valves from hearts explanted at the time of cardiac transplantation $(\mathrm{n}=6)$ were studied by immunostaining for interleukin-1 receptor antagonist. Aortic valve interstitial cells were isolated from valves, and receptor antagonist levels were determined from cell lysates (enzyme-linked immunosorbent assay). Osteogenic phenotype changes in valve cells stimulated by tolllike receptors 2 and 4 were determined by immunoblotting for bone morphogenetic protein- 2 after treatment with and without interleukin-1 receptor antagonist $(100 \mu \mathrm{g} / \mathrm{mL})$. Statistics were by analysis of variance.
\end{abstract}

Results: Interleukin-1 receptor antagonist was abundant in nonstenotic aortic valve leaflets and virtually absent in leaflets from stenotic valves. Aortic valve interstitial cells from grossly normal leaflets produced significantly more receptor antagonist at baseline and in response to toll-like receptor 2 and 4 stimulation, than did cells from diseased valves $(P<0.05)$. Interleukin-1 receptor antagonist was able to significantly attenuate toll-like receptor 2 , but not toll-like receptor 4 , stimulated bone morphogenetic protein-2 production in aortic valve interstitial cells $(P<.05)$.

Conclusions: Interleukin-1 receptor antagonist-mediated mechanisms of anti-inflammation are dysfunctional in stenotic valves. We conclude that such impaired mechanisms of anti-inflammation may contribute to the pathogenesis of aortic stenosis. (J Thorac Cardiovasc Surg 2011;141:481-6)

Calcific aortic stenosis has traditionally been considered a "degenerative" process with passive accumulation of calcium on the aortic valve leaflets. However, recent studies now suggest that calcific aortic stenosis may in fact be an active disease process; mechanisms of inflammation and osteogenesis appear to play important roles in the pathogenesis of aortic stenosis. ${ }^{1-6}$ As such, aortic stenosis may be an inflammatory disease.

The aortic valve interstitial cell (AVIC) has been implicated in the pathogenesis of aortic stenosis. ${ }^{7}$ In response to proinflammatory stimulation via toll-like receptors (TLRs) 2 and 4, the phenotype of human AVICs changes from that of a myofibroblast to that of a bone-forminglike cell. ${ }^{7-10}$ Characteristics of this osteogenic phenotype include an increased expression of the potent bone-

From the Division of Cardiothoracic Surgery, the University of Colorado, Aurora, Colo.

Disclosures: Authors have nothing to disclose with regard to commercial support.

Read at the 36th Annual Meeting of The Western Thoracic Surgical Association,

Ojai, California, June 23-26, 2010.

Received for publication May 28, 2010; revisions received Sept 30, 2010; accepted for publication Nov 2, 2010.

Address for reprints: David A. Fullerton, Head, Cardiothoracic Surgery, Box C-310, 12631 E. 17th Ave, Room 6602, PO Box 6511, Aurora, CO 80045 (E-mail: David. Fullerton@UCDenver.edu).

$0022-5223 / \$ 36.00$

Copyright (c) 2011 by The American Association for Thoracic Surgery

doi: $10.1016 /$ j.jtcvs.2010.11.002 forming protein, bone morphogenetic protein-2 (BMP-2), the osteogenic transcription factor, Runx 2 , and an increased expression and activity of alkaline phosphatase. ${ }^{7-10}$

The net inflammatory state of any tissue is determined by the relative balance of proinflammatory and antiinflammatory mechanisms. ${ }^{11}$ In response to proinflammatory stimulation, a deficiency of anti-inflammatory mechanisms will lead to unopposed actions of proinflammatory mechanisms. Such an imbalance of proinflammatory and anti-inflammatory mechanisms has been implicated in the pathogenesis of many inflammatory diseases. Specifically, increased tissue levels of interleukin- 1 beta (IL-1 $\beta$ ) relative to its anti-inflammatory antagonist, interleukin-1 receptor antagonist (IL-1RA), have been implicated in the pathogenesis of rheumatoid arthritis, inflammatory bowel disease, atherosclerosis, and other inflammatory diseases. ${ }^{12}$

IL- $1 \beta$ has recently been implicated in the pathogenesis of aortic stenosis as well. ${ }^{13}$ Produced by circulating mononuclear cells, the proinflammatory actions of IL- $1 \beta$ are mediated by the membrane-bound IL-1 receptor (IL-1R). ${ }^{14}$ The specific antagonist of IL- $1 \beta$ is the anti-inflammatory cytokine, IL-1RA, which blocks the actions of IL- $1 \beta$ at its receptor. ${ }^{12}$ The balance between IL-1 and IL-1RA in a given tissue may determine the development of inflammatory disease; a deficiency of the anti-inflammatory actions of IL-1RA relative to IL- $1 \beta$ leads to inflammation and tissue 


$$
\begin{aligned}
& \text { Abbreviations and Acronyms } \\
& \text { AVIC }=\text { aortic valve interstitial cell } \\
& \text { BMP-2 }=\text { bone morphogenetic protein- } 2 \\
& \text { ELISA }=\text { enzyme-linked immunosorbent assay } \\
& \text { IL-1 } \beta=\text { interleukin-1 beta } \\
& \text { IL-1R }=\text { IL-1 receptor } \\
& \text { IL-1RA }=\text { interleukin- } 1 \text { receptor antagonist } \\
& \text { LPS }=\text { lipopolysaccharide } \\
& \text { PBS }=\text { phosphate-buffered saline } \\
& \text { PGN }=\text { peptidoglycan } \\
& \text { TLR }=\text { toll-like receptor }
\end{aligned}
$$

destruction. ${ }^{12}$ Given that aortic stenosis appears to be an inflammatory disease and that a deficiency of IL-1RA has been implicated in the pathogenesis of many inflammatory diseases, we hypothesized that a deficiency of IL-1RA may contribute to the pathogenesis of aortic stenosis as well. We further hypothesized that the source of IL-1RA in aortic valve leaflets is the AVIC.

In nonstenotic and stenotic aortic valve leaflets, the purposes of this study were as follows: (1) to characterize IL-1RA in aortic valve leaflets, (2) to examine the production of IL-1RA by AVICs, and (3) to examine the ability of IL-1RA to prevent the osteogenic phenotype changes of AVICs induced by the proinflammatory activation of TLR-2 and TLR-4. The results of this study demonstrate the following: (1) IL-1RA is deficient in stenotic aortic valve leaflets; (2) production of IL-1RA is significantly reduced in AVICs from stenotic aortic valves; and (3), IL-1RA attenuates the osteogenic phenotypic change of AVICs induced by proinflammatory stimulation by TLR-2. These findings have not previously been described and provide mechanistic insight into the pathogenesis of aortic stenosis.

\section{MATERIALS AND METHODS}

This study was approved by the Colorado Multiple Institutional Review Board of the University of Colorado Health Sciences Center. All patients provided written informed consent.

\section{Cell Isolation and Culture}

Stenotic aortic valves were obtained from 6 patients who underwent aortic valve replacement surgery for calcific aortic stenosis (men, aged 54-77 years). Controls were nonstenotic aortic valves obtained from the explanted hearts of patients undergoing heart transplantation at the University of Colorado Hospital $(n=6)$. The etiology of heart failure was idiopathic dilated cardiomyopathy in all patients (men, aged 38-55 years). None of these patients was a previous ventricular assist device recipient. On gross examination, nonstenotic valve leaflets were thin and pliable, and their surfaces were smooth; they were grossly normal. Microscopic examination of hematoxylin and eosin-stained cryosections confirmed the absence of leukocytic infiltration. All stenotic valves were thick and exhibited overt calcification. Noncalcified tissue was used for cell isolation. Small sections of each valve were frozen in OCT solution (Triangle Biomedical Science, Durham, NC), placed in 10\% formaldehyde for histologic analysis, flash frozen for future tissue homogenization, or used for AVIC isolation. Isolation was by collagenase digestion, as previously described, ${ }^{2}$ and culture was in growth medium (Medium 199 [Lonza Walkersville, Inc, Walkersville, Md] with amphotericin B, penicillin G, streptomycin, and $10 \%$ fetal bovine serum) in an incubator supplied with $5 \%$ carbon dioxide. Cells of passages 3 to 6 were grown to $70 \%$ to $90 \%$ confluence and subcultured to 24-well cell culture plates for experiments. Cells of each patient were maintained as independent cultures.

\section{Chemicals and Reagents}

Medium 199 was purchased from Lonza (Walkersville, Md). Rabbit polyclonal antibody against human BMP-2 was obtained from ProSci (Poway, Calif). Mouse affinity purified antibody against human IL-1RA was obtained from eBioscience (San Diego, Calif). Human IL-1RA DuoSet ELISA kit was obtained from R\&D Systems (Minneapolis, Minn). Recombinant human IL-1RA was a generous gift from Dr Charles Dinarello (Aurora, Colo). Collagenase and other reagents were purchased from Sigma Chemical Co (St Louis, Mo).

\section{AVIC Treatment}

AVICs studied by enzyme-linked immunosorbent assay (ELISA) for IL1RA were serum-starved for 24 hours after reaching approximately $90 \%$ confluence, treated with serum-free medium alone (controls), lipopolysaccharide (LPS, $200 \mathrm{ng} / \mathrm{mL}$ ), and peptidoglycan (PGN, $10 \mu \mathrm{g} / \mathrm{mL}$ ). After 24 hours, the cells were washed once with cold phosphate-buffered saline (PBS) and cell lysates were prepared with $200 \mu \mathrm{L}$ of mammalian protein extraction reagent (Thermo Scientific, Rockford, Ill), centrifuged for 10 minutes at 10,000 RPM, and supernatant collected. AVICs treated with recombinant IL-1RA $(100 \mu \mathrm{g} / \mathrm{mL})$ were pretreated in serum-free medium alone (controls) or IL-1RA 1 hour before treatment with LPS or PGN (200 ng/mL and $10 \mu \mathrm{g} / \mathrm{mL}$, respectively). Cells were washed once with cold PBS and lysed with mammalian protein extraction reagent.

\section{Immunohistochemistry}

Immunohistochemical detection of IL-1RA in aortic valve leaflets was performed by the biotin-linked peroxidase technique. Cryosections $(5 \mu \mathrm{m})$ were prepared and dried at room temperature for 2 hours. Sections were fixed with $4 \%$ paraformaldehyde in PBS at room temperature for 10 minutes and then washed with PBS. Unless indicated, all incubations were performed at room temperature. Endogenous peroxidase activity was quenched by incubating sections with $0.5 \%$ hydrogen peroxide in methanol for 10 minutes. Sections were incubated for 1 hour with $10 \%$ goat serum in PBS to block nonspecific binding sites. Sections were then incubated overnight at $4^{\circ} \mathrm{C}$ with mouse affinity purified antibody against IL-1RA $(10 \mu \mathrm{g} / \mathrm{mL}$ in PBS containing $10 \%$ goat serum), followed by a 60 -minute incubation with biotinylated goat anti-mouse immunoglobulin G (1:200 dilution with PBS containing 10\% goat serum) from the Mouse ABC Staining System (Santa Cruz Biotechnology, Santa Cruz, Calif). Subsequently, sections were washed 3 times with PBS and incubated with avidin-biotin-peroxidase complex (1:50 dilution with PBS) for 30 minutes. After thorough washes with PBS, color development was carried out with peroxidase substrate $(0.03 \%$ $\mathrm{H}_{2} \mathrm{O}_{2}$ and $0.05 \%$ diaminobenzidine in double-distilled $\mathrm{H}_{2} \mathrm{O}$ ). Sections were counterstained with hematoxylin and eosin.

\section{Cytokine Assay}

IL-1RA levels in cell lysates and culture media were determined using a DuoSet ELISA kit per manufacturer's instructions.

\section{Immunoblotting}

Cell lysates were separated on $4 \%$ to $20 \%$ minigels and transferred onto nitrocellulose membranes (Bio-Rad Laboratories, Hercules, Calif). After blocking with $5 \%$ fat-free milk solution, BMP-2 was detected using primary antibodies to BMP-2 and then a corresponding peroxidase-linked 

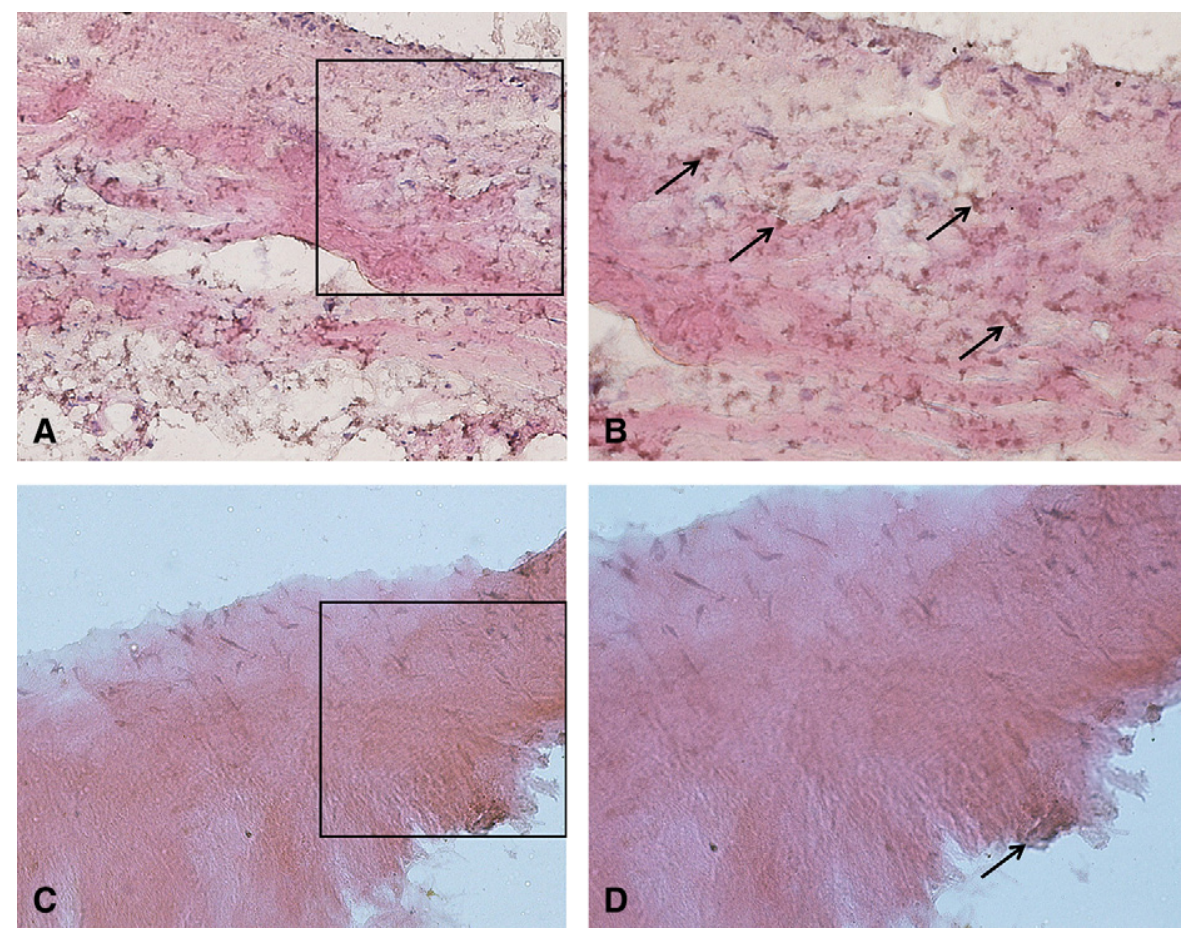

FIGURE 1. Immunohistochemical staining of sections of both nonstenotic and stenotic aortic valves for interleukin-1 receptor antagonist (IL-1RA). A and B, Nonstenotic aortic valve section in low power (A) and high power (B) that have been immunostained for IL-1RA (brown). The arrows show intense positive stains throughout both the lamina fibrosa and spongiosum. C and D, Stenotic aortic valve section in low (C) and high power (B). The section is intensely fibrotic and single positive stain in panel $\mathrm{D}$ is localized to a leukocyte in the lamina spongiosum.

secondary antibody (Cell Signaling Technology, Danvers, Mass) diluted 1:5000. Blots were developed with electrochemoluminescent reagent and exposed on x-ray film. Films were scanned, and Image $\mathbf{J}$ software was used to quantitate band density.

\section{Statistical Analysis}

Data are presented as mean \pm standard error and compared and analyzed by analysis of variance with a post-hoc Fisher exact test.

\section{RESULTS}

\section{Stenotic Aortic Valves Have Lower IL-1RA Levels Than Nonstenotic Valves}

Sections of stenotic aortic valves showed marked reduction in IL-1RA expression from nonstenotic aortic valves by immunohistochemistry. Microscopic examination of hematoxylin and eosin-stained cryosections showed stenotic valves were fibrotic and exhibited overt calcification. Nonstenotic valves were thin and had no evidence of fibrosis or calcification. Immunohistochemical staining of nonstenotic valves was positive diffusely for IL-1RA both in the lamina fibrosa and spongiosum (Figure 1, $A$ and $B$ ). Stenotic aortic valves had considerable fibrosis and staining for IL-1RA was virtually absent (Figure 1, $C$ and $D$ ).

\section{AVICs From Nonstenotic Aortic Valves Have Greater IL-1RA Levels Than AVICs From Stenotic Valves}

IL-1RA was significantly decreased in AVICs from stenotic aortic valves $(n=6)$ versus nonstenotic valves $(\mathrm{n}=6)$. IL-1RA levels were undetectable in lysates from untreated AVICs from stenotic valves compared with $710.2 \pm 213.6 \mathrm{pg} / \mathrm{mL}(P<.05)$ in nonstenotic AVICs. Production of IL-1RA in response to TLR stimulation was significantly less in AVICs from stenotic valves (Figure 2). In samples that were treated with proinflammatory stimulation of TLR-2 and TLR-4 with PGN and LPS, respectively, IL1RA levels in AVICs from stenotic aortic valves were 114.9 $\pm 86.5 \mathrm{pg} / \mathrm{mL}$ and $244.9 \pm 112.4 \mathrm{pg} / \mathrm{mL}$, respectively. Levels in nonstenotic AVICs after TLR-2 and TLR-4 stimulation were $1570.1 \pm 339.1 \mathrm{pg} / \mathrm{mL}$ and $1122.6 \pm$ $181.8 \mathrm{pg} / \mathrm{mL}$, respectively. Overall, there was a $6-$ and 10 -fold increase in IL-1RA in nonstenotic valve cells over stenotic cells after TLR-2 and TLR-4 activation, respectively.

\section{IL-1RA Treatment of AVICs Decreased BMP-2 Production}

Treatment of AVICs with recombinant IL-1RA decreased the production of BMP-2 and attenuated the change to an osteogenic phenotype ( $n=4$, Figure 3 ). After 24 hours of treatment with IL-1RA at $100 \mu \mathrm{g} / \mathrm{mL}$, densitometric analysis of cell lysates from immunoblots showed no significant change in expression of BMP-2 in unstimulated cells $(P<.05)$. Stimulation of TLR-2 (PGN) in AVICs from nonstenotic valves produced an $81.0 \% \pm 9.0 \%$ increase in BMP- 2 that was prevented by IL-1RA. However, stimulation of TLR-4 


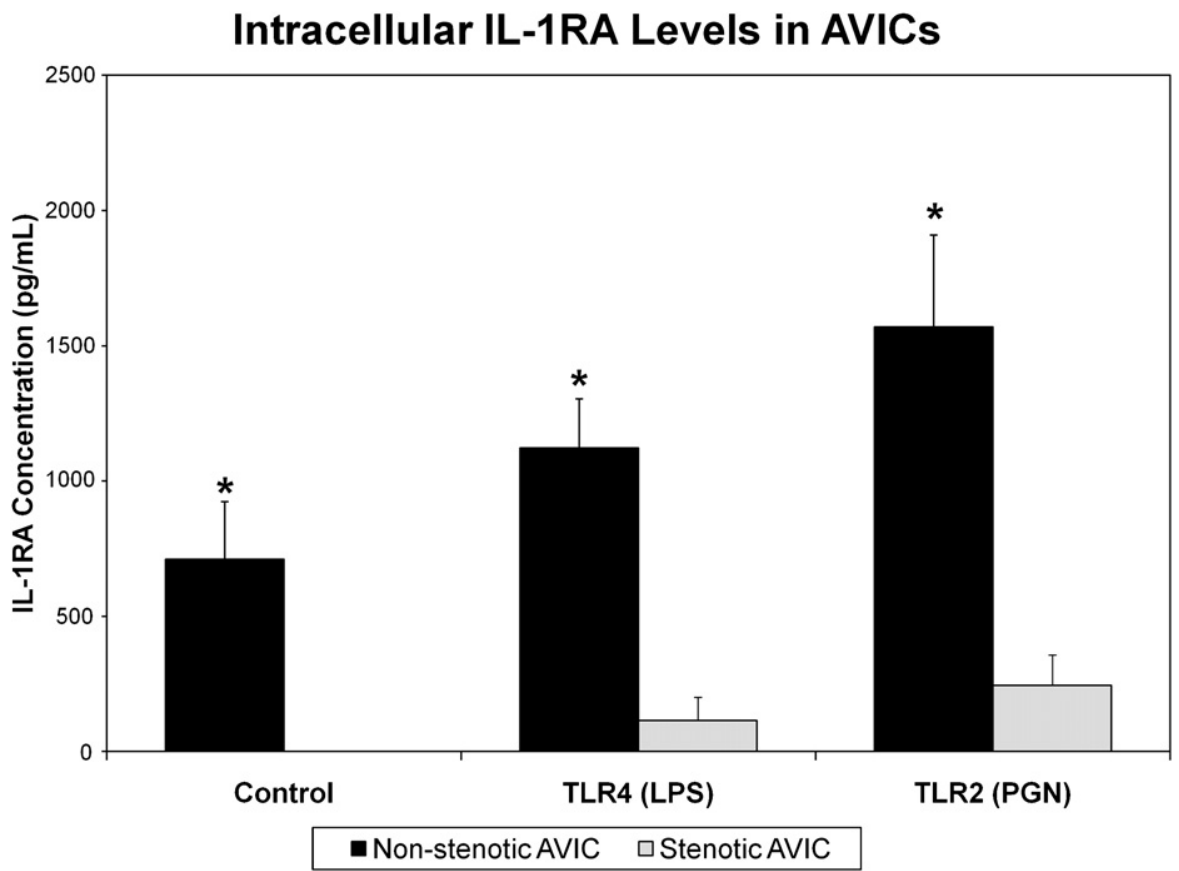

FIGURE 2. Intracellular interleukin-1 receptor antagonist (IL-1RA) in cell lysates of nonstenotic aortic valve interstitial cells (AVICs) and those from stenotic AVICs. IL-1RA levels were undetectable in AVICs from unstimulated (control) stenotic aortic valves versus their nonstenotic counterparts. After activation of toll-like receptor $4(T L R-4)$ and toll-like receptor $2(T L R-2)$ with lipopolysaccharide $(L P S)$ and peptidoglycan $(P G N)$, respectively, there were 1.58-fold and 2.21-fold increases in IL-1RA in nonstenotic AVICs over unstimulated cells (control). In response to TLR-2 and TLR-4 stimulation, IL-1RA production was significantly less in AVICs from stenotic valves compared with nonstenotic valves. ${ }^{*} P<.05$, stenotic AVICs versus nonstenotic AVICs.

(LPS) likewise produced a significant increase in BMP-2 production that was not affected by IL-1RA.

\section{DISCUSSION}

The results of the present study demonstrate that an important mechanism of anti-inflammation is dysfunctional in aortic stenosis. Although nonstenotic aortic valve leaflets were found to have an abundance of IL-1RA, it was virtually absent in leaflets from stenotic aortic valves. Aortic valve interstitial cells were found to be an important source of IL-1RA in nonstenotic aortic valves and demonstrated significantly increased production in response to proinflammatory stimulation. Conversely, IL-1RA production by AVICs from stenotic aortic valves was significantly less than in controls. Further, the potential role of IL-1RA as an important anti-inflammatory defense mechanism was demonstrated; the exogenous administration of IL-1RA significantly attenuated the phenotypic changes induced by proinflammatory stimulation of TLR-2. These data suggest that impaired mechanisms of anti-inflammation, and specifically a deficiency of IL-1RA, are associated with the pathogenesis of calcific aortic stenosis. To our knowledge, these findings have not previously been described.

In the present study, isolated AVICs were examined in vitro. A limitation of any such study is that the behavior of cells in vitro may differ from the behavior of those in vivo. However, we have previously reported that human
AVICs that have been passaged multiple times have function comparable with those of freshly isolated cells. ${ }^{7}$ A second limitation of the present study is that it is not possible to know whether the deficiency of the levels and production of IL-1RA in stenotic valves preceded the clinical manifestation of aortic stenosis in the valves in vivo. Nonetheless, the finding has important implications.

IL- $1 \beta$ has been implicated in the pathogenesis of many inflammatory diseases, including aortic stenosis. ${ }^{5,13} \mathrm{At}$ the tissue level, the net balance between the levels of IL$1 \beta$ and IL-1RA helps determine whether inflammatory disease develops with its associated structural damage; local overproduction of IL-1 $\beta$ and/or deficiency of IL-1RA may lead to inflammatory disease. Inflammatory diseases such as rheumatoid arthritis and inflammatory bowel disease, among others, are thought to arise from a deficiency of IL-1RA. ${ }^{12}$ In fact, the administration of recombinant IL-1RA is an effective therapy for rheumatoid arthritis.

Secretory and intracellular isoforms of IL-1RA have been identified in humans. Secretory IL-1RA is produced and secreted by monocytes, neutrophils and macrophages. Intracellular IL-1RA has been identified in hepatocytes, macrophages, fibroblasts, endothelial cells, and vascular smooth muscle cells. ${ }^{12}$ However, IL-1RA has not previously been described in human aortic valves, nor has its production by AVICs been previously characterized. It is not possible to know from the present study whether the 


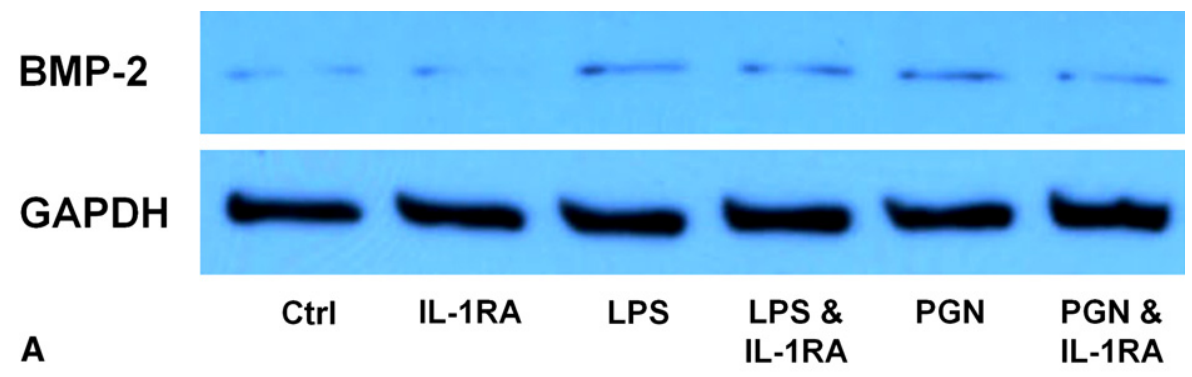

BMP-2 Expression in AVICs Treated with IL-1RA

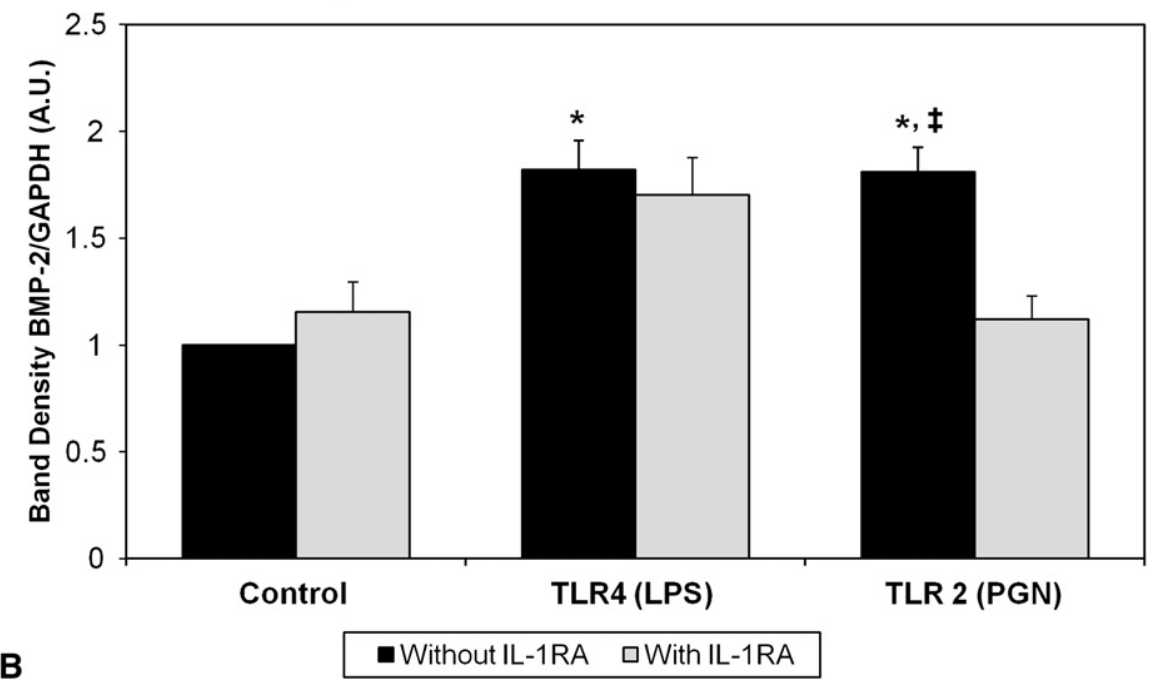

FIGURE 3. Bone morphogenetic protein-2 (BMP-2) expression in nonstenotic aortic valve interstitial cells (AVICs) treated with proinflammatory stimulation and interleukin-1 receptor antagonist (IL-1RA). A Representative immunoblot of lysates of AVICs treated with lipopolysaccharide (LPS) and peptidoglycan $(P G N)$ after pretreatment with IL-1RA. B, Densitometric values for BMP-2 after activation of toll-like receptor 4 (TLR-4) and toll-like receptor-2 (TLR-2) with LPS or PGN, respectively. TLR-2-stimulated BMP-2 production was blocked by IL-1RA. However, TLR-4-stimulated BMP-2 production was unaffected. $* P<.05$ versus control. $\ddagger P<.05$ IL-1RA treatment versus untreated, before PGN treatment.

deficiency of IL-1RA found in stenotic aortic valves preceded the clinical development of aortic stenosis in vivo. However, the results of the present study are consistent with findings in other inflammatory diseases in which a deficiency of IL-1RA has been implicated and suggest that diminished levels of IL-1RA may contribute to the pathogenesis of human aortic stenosis Further, Isoda and colleagues ${ }^{15}$ recently demonstrated echocardiographic evidence of aortic stenosis and histologic evidence of increased valve thickness, inflammatory infiltrates, and calcification in mice lacking IL-1RA.

$\mathrm{We}^{7-10}$ have previously demonstrated that isolated AVICs acquire an osteogenic phenotype when activated by proinflammatory stimuli mediated by TLR-2 and -4. An important characteristic of this osteogenic phenotype is the production of the bone-forming protein, BMP-2. A novel finding of the present study was the inhibition by IL-1RA of TLR-2-induced BMP-2 expression in AVICs. It did not affect TLR-4-induced BMP-2 expression. Inhibition of TLR-2 stimulation by IL-1RA has not previously been described. Likewise, there has been no reported interaction of TLR-2 with the IL-1R, and we ${ }^{7}$ have previously reported that isolated human AVICs do not produce IL- $1 \beta$ in response to TLR-2 or -4 stimulation. Heretofore, the only known action of IL-1RA has been inhibition of the IL-1R, and for that reason, it is considered a specific antagonist of IL- $1 \beta$. This finding of the present study, however, implies that the anti-inflammatory actions of IL-1RA may be mediated by mechanisms in addition to its inhibition of IL-1R. Further elucidation of this IL-1RA inhibition of TLR-2 stimulation requires further investigation and was beyond the scope of the present study. Nonetheless, it highlights the potential role of IL-1RA as an important antiinflammatory defense mechanism in the aortic valve.

In summary, the results of the present study demonstrated a marked deficiency of an important anti-inflammatory cytokine in aortic stenosis. Although IL-1RA was found throughout nonstenotic aortic valve leaflets, it was virtually absent in leaflets from stenotic aortic valves. Further, the levels of intracellular IL-1RA were undetectable in stenotic AVICs from stenotic valves and were significantly lower after proinflammatory stimulation than in controls. The 
importance of IL-1RA as an anti-inflammatory defense mechanism in AVICs was demonstrated by its attenuation of TLR-2-induced osteogenic phenotype changes. Previous work has implicated mechanisms of inflammation in the pathogenesis of aortic stenosis. The results of the present study provide additional insight into this pathogenesis and suggest that dysfunctional protective mechanisms of antiinflammation may play a role.

\section{References}

1. Rajamannan NM, Subramaniam M, Rickard D, Stock SR, Donovan J, Springett M, et al. Human aortic valve calcification is associated with an osteoblast phenotype. Circulation. 2003;107:2181-4.

2. Anger T, Carson W, Weyand M, Daniel WG, Hoeher M, Garlichs CD. Atherosclerotic inflammation triggers osteogenic bone transformation in calcified and stenotic human aortic valves: still a matter of debate. Exp Mol Pathol. 2009;86:10-7.

3. Mohler ER 3rd, Gannon F, Reynolds C, Zimmerman R, Keane MG, Kaplan FS. Bone formation and inflammation in cardiac valves. Circulation. 2001;103: 1522-8.

4. Kaden JJ, Dempfle CE, Grobholz R, Fischer CS, Vocke DC, Kilic R, et al. Inflammatory regulation of extracellular matrix remodeling in calcific aortic valve stenosis. Cardiovasc Pathol. 2005;14:80-7.

5. Kaden JJ, Kilic R, Sarikoc A, Hagl S, Lang S, Hoffmann U, et al. Tumor necrosis factor alpha promotes an osteoblast-like phenotype in human aortic valve myofibroblasts: a potential regulatory mechanism of valvular calcification. Int J Mol Med. 2005;16:869-72.
6. Naito Y, Tsujino T, Wakabayashi K, Matsumoto M, Ohyanagi M, Mitsuno M, et al. Increased interleukin-18 expression in nonrheumatic aortic valve stenosis. Int J Cardiol. 2010;144:260-3. Epub 2009 Feb 13.

7. Meng X, Ao L, Song Y, Babu A, Yang X, Wang M, et al. Expression of functional toll-like receptors 2 and 4 in human aortic valve interstitial cells: potential roles in aortic valve inflammation and stenosis. Am J Physiol Cell Physiol. 2008;294: C29-35.

8. Yang X, Fullerton DA, Su X, Ao L, Cleveland JC Jr, Meng X. Pro-osteogenic phenotype of human aortic valve interstitial cells is associated with higher levels of toll-like receptors 2 and 4 and enhanced expression of bone morphogenetic protein 2. J Am Coll Cardiol. 2009;53:491-500.

9. Babu AN, Meng X, Zou N, Yang X, Wang M, Song Y, et al. Lipopolysaccharide stimulation of human aortic valve interstitial cells activates inflammation and osteogenesis. Ann Thorac Surg. 2008;86:71-6.

10. Yang X, Meng X, Su X, Mauchley DC, Ao L, Cleveland JC Jr, et al. Bone morphogenic protein 2 induces Runx 2 and osteopontin expression in human aortic valve interstitial cells: role of Smad1 and extracellular signal-regulated kinase 1/2. J Thorac Cardiovasc Surg. 2009;138:1008-15.

11. Opal SM, DePalo VA. Anti-inflammatory cytokines. Chest. 2000;117:1162-72.

12. Arend WP. The balance between IL-1 and ilL-1Ra in disease. Cytokine Growth Factor Rev. 2002;13:323-40.

13. Kaden JJ, Dempfle CE, Grobholz R, Tran HT, Kilic R, Sarikoc A, et al. Interleukin-1 beta promotes matrix metalloproteinase expression and cell proliferation in calcific aortic valve stenosis. Atherosclerosis. 2003;170:205-11.

14. Dinarello CA. Interleukin-1beta and the autoinflammatory diseases. $\mathrm{N} \mathrm{Engl}$ J Med. 2009;360:2467-70.

15. Isoda K, Matsuki T, Kondo H, Iwakura Y, Ohsuzu F. Deficiency of interleukin-1 receptor antagonist induces aortic valve disease in BALB/c mice. Arterioscler Thromb Vasc Biol. 2010;30:708-15. 